\title{
A NEW CLASS OF GENERATING FUNCTIONS FOR HYPERGEOMETRIC POLYNOMIALS
}

\author{
DAVID ZEITLIN
}

\begin{abstract}
A new class of generating functions for generalized hypergeometric polynomials is obtained. Applications are given for the classical polynomials containing parameters, such as Jacobi, ultraspherical, and Laguerre polynomials, as well as for Hermite and Bessel polynomials. Two classes of generating functions recently obtained by $\mathrm{J}$. W. Brown are discussed for independence.
\end{abstract}

1. Introduction. Recently, Brown [3] proved the following

Lemma. Given a sequence $\phi_{n}(n \geqq 0)$, define the new one

$$
\psi_{n}=\sum_{k=0}^{n}\left(\begin{array}{c}
a+b n \\
n-k
\end{array}\right) \phi_{k} \quad(n \geqq 0) .
$$

Then

$$
\sum_{n=0}^{\infty} \psi_{n}\left[\frac{x}{(1+x)^{b}}\right]^{n}=\frac{(1+x)^{1+a}}{1+(1-b) x} \sum_{n=0}^{\infty} \phi_{n} x^{n}
$$

and

$$
\sum_{n=0}^{\infty} \frac{a}{a+b n} \psi_{n}\left[\frac{x}{(1+x)^{b}}\right]^{n}=(1+x)^{a} \sum_{n=0}^{\infty} \frac{a}{a+b n} \phi_{n} x^{n} .
$$

Recalling that $(a)_{k}=a(a+1) \cdots(a+k-1)$ and defining

$$
\phi_{k}=\frac{\left(a_{1}\right)_{k} \cdots\left(a_{p}\right)_{k}}{\left(b_{1}\right)_{k} \cdots\left(b_{q}\right)_{k}} \cdot \frac{(-x)^{k}}{k !},
$$

where the $a_{i}$ and $b_{i}$ are independent of $n$, we obtain from (1.1) the generalized hypergeometric polynomial

$$
R_{n}^{(a, b)}(x) \equiv \psi_{n}=\left(\begin{array}{c}
a+b n \\
n
\end{array}\right)_{p+1} F_{q+1}\left[\begin{array}{r}
-n, a_{1}, \cdots, a_{p} ; x \\
1+a+(b-1) n, b_{1}, \cdots, b_{q} ;
\end{array}\right]
$$

The two generating functions, (1.2) and (1.3), were applied in [3] only

Received by the editors September 2, 1969.

AMS Subject Classifications. Primary 3320, 3340, 4010.

Key Words and Phrases. Generating functions, generalized hypergeometric polynomials, Jacobi polynomials, ultraspherical polynomials, Laguerre polynomials, Bessel polynomials, Hermite polynomials. 
for $b=\frac{1}{2}$, a value that is too restrictive for complete generality (see Brown [2]).

The proof of (1.2) and (1.3), respectively, is based on the following two well-known identities

$$
\begin{aligned}
& \sum_{n=0}^{\infty}\left(\begin{array}{c}
a+b n \\
n
\end{array}\right)\left[\frac{x}{(1+x)^{b}}\right]^{n}=\frac{(1+x)^{1+a}}{1+(1-b) x}, \\
& \sum_{n=0}^{\infty} \frac{a}{a+b n}\left(\begin{array}{c}
a+b n \\
n
\end{array}\right)\left[\frac{x}{(1+x)^{b}}\right]^{n}=(1+x)^{a},
\end{aligned}
$$

which occur as special cases of (1.2) and (1.3), respectively, when $\phi_{0}=1$ and $\phi_{n}=0$ for $n \geqq 1$. Pólya and Szegö [7, pp. 301-302] obtained (1.6) and (1.7) by means of Lagrange's theorem; and recently, Gould [5] derived them by direct summation.

We now point out that the pair of identities (1.6) and (1.7) are not independent, i.e., either one implies the other. Thus, we now state and prove

Lemma 1. A necessary and sufficient condition for (1.7) is (1.6).

Proof. To show that (1.6) implies (1.7), one uses the identity

$$
\frac{(a+b n)}{(a+b n)}\left(\begin{array}{c}
a+b n \\
n
\end{array}\right)=\frac{a}{a+b n}\left(\begin{array}{c}
a+b n \\
n
\end{array}\right)+b\left(\begin{array}{c}
a+b n-1 \\
n-1
\end{array}\right) .
$$

This portion of the proof appears in the recent book by Riordan [8, p. 148].

To show that (1.7) implies (1.6), set $y=x /(1+x)^{b}$ in (1.7). Noting that $d y / d x=(1+(1-b) x) /(1+x)^{b+1}$, one differentiation of (1.7) with respect to $x$, with $a$ replaced by $a+1-b$, yields (1.6). This portion of the proof appears in the recent paper by Carlitz [4, p. 825]. This completes the proof of Lemma 1.

We now note that the pair of identities, (1.2) and (1.3), which required (1.6) and (1.7) in their proof, are also not independent. Thus, we now state

Lemma 2. A necessary and sufficient condition for (1.3) is (1.2).

The proof of Lemma 2 is given in $\$ 4$ below.

Taking (1.2), with (1.5), as a basic, first class of new generating functions, we now proceed to obtain a second class of new generating functions which are independent of the first class.

2. A new generating function. Recently, Gould [6, p. 196] proved the following identity: 


$$
\begin{aligned}
& \sum_{j=0}^{\infty} \frac{c}{c+b j}\left(\begin{array}{c}
a+b j \\
j
\end{array}\right)\left[\frac{x}{(1+x)^{b}}\right]^{j} \\
& \quad=(1+x)^{a} \sum_{n=0}^{\infty}(-1)^{n}\left(\begin{array}{c}
a-c \\
n
\end{array}\right)\left(\begin{array}{c}
(c / b)+n \\
n
\end{array}\right)^{-1} \cdot\left[\frac{x}{1+x}\right]^{n} .
\end{aligned}
$$

For $c=a,(2.1)$ reduces to (1.7). Formally, the limit of (2.1), as $c \rightarrow \infty$, is (1.6). Thus, for finite $c, c \neq a,(2.1)$ is independent of (1.6). As an application of (2.1), the independent, companion identity to (1.2) is now given by

THEOREM 1. Let $\phi_{n}$ and $\psi_{n}$ be defined by (1.1). Choose $c$ so that $(a-c)$ is a positive integer. Then

$$
\begin{aligned}
\sum_{n=0}^{\infty} & \frac{c}{c+b n} \psi_{n}\left[\frac{x}{(1+x)^{b}}\right]^{n} \\
= & (1+x)^{a} \sum_{n=0}^{a-c}(-1)^{n}\left(\begin{array}{c}
a-c \\
n
\end{array}\right)\left(\frac{x}{1+x}\right)^{n} \\
& \cdot\left\{\sum_{k=0}^{\infty} \frac{c}{c+b k}\left(\begin{array}{c}
(c / b)+k+n \\
n
\end{array}\right)^{-1} \phi_{k} x^{k}\right\} .
\end{aligned}
$$

Proof. Let $L$ denote the left-hand side of (2.2). Substitution by (1.1) in $L$ gives

$$
\begin{aligned}
L=\sum_{k=0}^{\infty} & \frac{c}{c+b k} \phi_{k}\left[\frac{x}{(1+x)^{b}}\right]^{k} \\
& \cdot\left\{\sum_{j=0}^{\infty} \frac{(c+b k)}{(c+b k)+b j}\left(\begin{array}{c}
a+b k+b j \\
j
\end{array}\right)\left[\frac{x}{(1+x)^{b}}\right]^{j}\right\} .
\end{aligned}
$$

Then, using (2.1) with $c$ replaced by $c+b k$ and $a$ replaced by $a+b k$ and noting that $a-c$ is a positive integer, we obtain (2.2). For $c=a$, (2.2) reduces to (1.3).

We now state our principal result.

THEOREM 2. A generating function for the generalized hypergeometric polynomials, $R_{n}^{(a, b)}(x)$, defined by (1.5), with $a-c$ as a positive integer, is given by

$$
\begin{array}{r}
{[1+g(t)]^{-a} \sum_{n=0}^{\infty} \frac{c}{c+b n} R_{n}^{(a, b)}(x) t^{n}=\sum_{n=0}^{a-c} A_{n}(a, b, c, t) \cdot{ }_{n+1+p} F_{n+1+q}} \\
\cdot\left[\begin{array}{c}
c / b, \cdots,(c / b)+n, a_{1}, \cdots, a_{p} ; \\
(c / b)+1, \cdots,(c / b)+1+n, b_{1}, \cdots, b_{q} ;
\end{array}\right]
\end{array}
$$


where $A_{0}(a, b, c, t)=1$,

$$
\begin{array}{r}
A_{n}(a, b, c, t)=n !(-1)^{n}\left(\begin{array}{c}
a-c \\
n
\end{array}\right)\left(\frac{g(t)}{1+g(t)}\right)^{n} \prod_{j=1}^{n} \frac{b}{c+j b} \\
(n=0,1, \cdots, a-c),
\end{array}
$$

and $g(t)$ is defined as the formal inverse of the function $h(t)=t /(1+t)^{b}$, i.e., $g(h(t))=h(g(t))=t$.

Proof. Set $x=g(t)$ in (2.2) to obtain

$$
\begin{aligned}
& {[1+g(t)]^{-a} \cdot \sum_{n=0}^{\infty} \frac{c}{c+b n} \psi_{n} t^{n}=\sum_{n=0}^{a-c} A_{n}^{*}(t)} \\
& \cdot \sum_{k=0}^{\infty} \frac{(c / b)}{(k+(c / b))(k+(c / b)+1) \cdots(k+(c / b)+n)} \phi_{k}(g(t))^{k},
\end{aligned}
$$

where $A_{n}^{*}(t)=A_{n}(a, b, c, t) / \prod_{j=1}^{n}(b /(c+j b))$. We define $\phi_{k}$ by (1.4) to obtain (1.5). Recalling (1.4) and noting that

$\frac{1}{k+(c / b)+j}=\frac{b}{c+j b}((c / b)+j)_{k} /((c / b)+j+1)_{k}, \quad j=0,1, \cdots, n$ we obtain (2.3) from (2.5).

3. New generating functions for classical polynomials. The Jacobi polynomials are given by

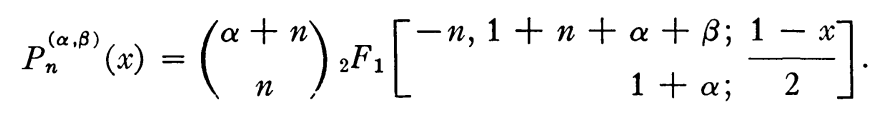

Recalling (1.5), replace in (3.1) $\alpha$ by $a+(b-1) n$, and $\beta$ by $\beta-b n$. Then, Theorem 2, with $p=1, a_{1}=1+a+\beta$, and $q=0$, gives the generating function

$$
\begin{aligned}
{[1+g(t)]^{-a} \cdot \sum_{n=0}^{\infty} \frac{c}{c+b n} P_{n}^{(a+(b-1) n, \beta-b n)}(x) t^{n} } \\
=\sum_{n=0}^{a-c} A_{n}(a, b, c, t) \cdot{ }_{n+2} F_{n+1} \\
\cdot\left[\begin{array}{c}
\left.c / b, \cdots,(c / b)+n, 1+a+\beta ; \frac{(x-1) g(t)}{2}\right] . \\
(c / b)+1, \cdots,(c / b)+1+n ;
\end{array}\right.
\end{aligned}
$$

The generating function for the ultraspherical polynomials is obtained from (3.2) by setting $a+(b-1) n=\beta-b n$, i.e., $\beta=a$ and $b=\frac{1}{2}$, and noting that for $b=\frac{1}{2}, g(t)=(t / 2)\left(t+\left(t^{2}+4\right)^{1 / 2}\right)$. 
We recall that Laguerre polynomials are given by

$$
L_{n}^{(\alpha)}(x)=\left(\begin{array}{c}
\alpha+n \\
n
\end{array}\right){ }_{1} F_{1}\left[\begin{array}{c}
-n ; \\
1+\alpha ;
\end{array}\right]
$$

Thus, for $p=q=0$, Theorem 2 gives the generating function

$$
\begin{aligned}
{[1+g(t)]^{-a} } & \cdot \sum_{n=0}^{\infty} \frac{c}{c+b n} L_{n}^{(a+(b-1) n)}(x) t^{n}=\sum_{n=0}^{a-c} A_{n}(a, b, c, t) \\
& \cdot{ }_{n+1} F_{n+1}\left[\begin{array}{c}
c / b, \cdots,(c / b)+n ; \\
(c / b)+1, \cdots,(c / b)+1+n ;
\end{array}-x g(t)\right] .
\end{aligned}
$$

Recently, Al-Salam [1] observed that the Bessel polynomials $y_{n}(x, a, 1)=n !(-1)^{n} x^{n} L_{n}^{(1-a-2 n)}(1 / x)$. Thus, (3.4) is readily modified to give a new generating function for Bessel polynomials.

Since $H_{2 n}(x)=n !(-1)^{n} 2^{2 n} L_{n}^{(-1 / 2)}\left(x^{2}\right)$ and

$$
H_{2 n+1}(x)=n !(-1)^{n} 2^{2 n+1} x L_{n}^{(1 / 2)}\left(x^{2}\right),
$$

where $H_{n}(x)$ are Hermite polynomials, we obtain from (3.3), $H_{2 n}(x)$ $=((2 n) ! / n !)(-1)^{n}{ }_{1} F_{1}\left[-n ; \frac{1}{2} ; x^{2}\right]$ and

$$
H_{2 n+1}(x)=((2 n+1) ! / n !)(-1)^{n}(2 x) \cdot{ }_{1} F_{1}\left[-n ; 3 / 2 ; x^{2}\right] .
$$

Thus, from Theorem 2 , with $p=q=0, b=1$, and $g(t)=t /(1-t)$, we obtain, respectively, for $a=-1 / 2$ (or $k=0$ ) and $a=1 / 2$ (or $k=1$ ), with $t$ replaced by $-4 t$ for simplification,

$$
\begin{array}{r}
(1+4 t)^{a} \sum_{n=0}^{\infty} \frac{c}{n !(c+n)} H_{2 n+k}(x) t^{n}=(2 x)^{k} \sum_{n=0}^{a-c} A_{n}(a, 1, c,-4 t) \\
\cdot_{n+1} F_{n+1}\left[\begin{array}{r}
\left.c, \cdots, c+n ; \frac{4 x^{2} t}{1+\cdots t}\right], \\
c+1, c+1+n ; 1+4 t
\end{array}\right] \\
\left(a=(-1)^{k+1} / 2, k=0,1\right) .
\end{array}
$$

We note that the choice of $b=1 / 2$ in [3] precluded applications for Hermite polynomials.

4. Proof of Lemma 2. To show that (1.3) implies (1.2), let $y$ $=x /(1+x)^{b}$ so that $(1 / y) d y / d x=(1+(1-b) x) /(x(1+x))$. Differentiation of (1.3) with respect to $x$, with simplification and multiplication by the factor $b$, gives 


$$
\begin{aligned}
\sum_{n=0}^{\infty} \frac{b n}{a+b n} \psi_{n} y^{n}= & \frac{(1+x)^{1+a}}{1+(1-b) x} \sum_{n=0}^{\infty} \phi_{n} x^{n} \\
& -(1+x)^{a} \cdot \sum_{n=0}^{\infty} \frac{a}{a+b n} \phi_{n} x^{n} .
\end{aligned}
$$

Substitution in (4.1), for the third sum, by (1.3) gives (1.2).

To show that (1.2) implies (1.3), let $S$ denote the series on the lefthand side of (1.3). We now rewrite (1.2) as

$$
\sum_{n=0}^{\infty} \psi_{n} y^{n}=S+T, \quad \text { where } T=\sum_{n=0}^{\infty} \frac{b n}{a+b n} \psi_{n} y^{n} .
$$

Using (1.1), we obtain

$$
\begin{aligned}
T= & \sum_{k=0}^{\infty} \phi_{k} \sum_{n=k}^{\infty} \frac{b n}{a+b n}\left(\begin{array}{c}
a+b n \\
n-k
\end{array}\right) y^{n} \quad(\text { set } n=k+i) \\
= & \sum_{k=0}^{\infty} \phi_{k} y^{k}\left\{\left(\begin{array}{c}
a+b k+b i \\
i
\end{array}\right) y^{i}\right. \\
& \left.\quad-\frac{a}{a+b k} \sum_{i=0}^{\infty} \frac{a+b k}{a+b k+b i}\left(\begin{array}{c}
a+b k+b i \\
i
\end{array}\right) y^{i}\right\} \\
= & \sum_{k=0}^{\infty} \phi_{k} y^{k}\left\{\frac{(1+x)^{1+a+b k}}{1+(1-b) x}-\frac{a}{a+b k}(1+x)^{a+b k}\right\} \\
= & \sum_{n=0}^{\infty} \psi_{n} y^{n}-(1+x)^{a} \cdot \sum_{k=0}^{\infty} \frac{a}{a+b k} \phi_{k} x^{k},
\end{aligned}
$$

having used (1.6) and (1.7) with $a$ replaced by $a+b k$. Substitution for $T$ in (4.2) by (4.3) gives (1.3). This completes the proof.

5. A generalization of Theorem 2. Our generalization of Theorem 1 is given by

Theorem 3. Choose $c$ so that $(a-c)$ is a positive integer. Then

$$
\begin{aligned}
\sum_{n=0}^{\infty} \frac{c}{c+b n} \psi_{n} *\left[\frac{x}{(1+x)^{b}}\right]^{n} \\
=(1+x)^{a} \sum_{n=0}^{a-c}(-1)^{n}\left(\begin{array}{c}
a-c \\
n
\end{array}\right)\left(\frac{x}{1+x}\right)^{n} \\
\cdot\left\{\sum_{k=0}^{\infty} \frac{c}{c+b m k}\left(\begin{array}{c}
(c / b)+m k+n \\
n
\end{array}\right)^{-1} \phi_{k}^{*} x^{m k}\right\},
\end{aligned}
$$


where

$$
\psi_{n}^{*}=\sum_{k=0}^{[n / m]}\left(\begin{array}{l}
a+b n \\
n-m k
\end{array}\right) \phi_{k}^{*} \quad(n \geqq 0 ; m=1,2, \cdots) .
$$

Proof. Apply the identity (5.3) to the left-hand side of (5.1), where

$$
\sum_{n=0}^{\infty} \sum_{k=0}^{[n / m]} B(n, k)=\sum_{k=0}^{\infty} \sum_{n=0}^{\infty} B(n+m k, k)
$$

then use (2.1) with $c$ replaced by $c+b m k$ and $a$ replaced by $a+b m k$.

If we define $\phi_{\boldsymbol{k}}^{*}=(-1)^{(m+1) k} \phi_{k}$ (see (1.4)), we obtain from (5.2) the generalized hypergeometric polynomial which reduces to (1.5) for $m=1$, namely,

$$
R_{n, m}^{(a, b)}(x) \equiv \psi_{n}^{*}=\left(\begin{array}{c}
a+b n \\
n
\end{array}\right) \cdot \sum_{k=0}^{[n / m]} \frac{(-n)_{m k}}{(1+a+(b-1) n)_{m k}}(-1)^{k} \phi_{k} .
$$

Our desired result is now obtained as an application of (5.1) and (5.4). Using the same notation of Theorem 2 where applicable, we now state

THEOREM 4. A generating function for the hypergeometric polynomials (5.4) is given by

$$
\begin{aligned}
{[1+g(t)]^{-a} } & \sum_{n=0}^{\infty} \frac{c}{c+b n} R_{n, m}^{(a, b)}(x) t^{n} \\
= & \sum_{n=0}^{a-c} A_{n}(a, b, c, t) \\
& \cdot{ }_{n+1+p} F_{n+1+q}\left[\begin{array}{l}
d_{0}, \cdots, d_{n}, a_{1}, \cdots, a_{p} ; \\
r_{0}, \cdots, r_{n}, b_{1}, \cdots, b_{q} ;
\end{array}(-g(t))^{m} x\right],
\end{aligned}
$$

where $d_{j}=(c+b j) /(m b)$ and $r_{j}=d_{j+m}, j=0,1, \cdots, n$. For the special case $c=a,(5.5)$ gives the recent result of $H . M$. Srivastava [9].

\section{REFERENCES}

1. W. A. Al-Salam, The Bessel polynomials, Duke Math. J. 24 (1957), 529-545. MR 19, 849.

2. J. W. Brown, New generating functions for classical polynomials, Notices Amer. Math. Soc. 16 (1969), 412. Abstract \#69T-B29.

3. - New generating functions for classical polynomials, Proc. Amer. Math. Soc. 21 (1969), 263-268. MR 38 \#4734.

4. L. Carlitz, Some generating functions for Laguerre polynomials, Duke Math. J. 35 (1968), 825-828. 
5. H. W. Gould, Some generalizations of Vandermonde's convolution, Amer. Math. Monthly 63 (1956), 84-91. MR 17, 702.

6. - A series transformation for finding convolution identities, Duke Math. J. 28 (1961), 193-202. MR 23 \#A1216.

7. G. Pólya and G. Szegö, Aufgaben und Lehrsätze aus der Analysis, Vol. I, 3rd ed., Die Grundlehren der math. Wissenschaften, Bände 19, 20, Springer-Verlag, Berlin and New York, 1964. MR 30 \#1219 a,b.

8. J. Riordan, Combinatorial identities, Wiley, New York, 1968. MR 38 \#53.

9. H. M. Srivastava, $A$ class of generating functions for generalized hypergeometric polynomials, Notices Amer. Math. Soc. 16 (1969), 975. Abstract \#69T-B198.

1650 Vincent Avenue North, Minneapolis, Minnesota 55411 